\title{
Visual stimulus-specific habituation of innate defensive behaviour in mice
}

\author{
Azadeh Tafreshiha, Sven A. van der Burg, Kato Smits, Laila A. Blömer and J. Alexander Heimel*
}

\begin{abstract}
Innate defensive responses such as freezing or escape are essential for animal survival. Mice show defensive behaviour to stimuli sweeping overhead, like a bird cruising the sky. Here, we tested this in young male mice and found that mice reduced their defensive freezing after sessions with a stimulus passing overhead repeatedly. This habituation is stimulus specific, as mice freeze again to a novel shape. Habituation occurs regardless of the visual field location of the repeated stimulus. The mice generalized over a range of sizes and shapes, but distinguished objects when they differed in both size and shape. Innate visual defensive responses are thus strongly influenced by previous experience as mice learn to ignore specific stimuli.
\end{abstract}

\section{KEY WORDS: Innate behaviour, Threat, Freezing, Defensive} behaviour

\section{INTRODUCTION}

Innate visual responses are observed across the animal kingdom. Animals instinctively freeze or flee when they spot a threat (De Franceschi et al., 2016; Eilam, 2005; Temizer et al., 2015; Yilmaz and Meister, 2013), and orient towards more attractive targets (Ewert, 1987; Hoy et al., 2016). Even human new-borns instinctively respond to looming objects (Orioli et al., 2018). Though these innate behaviours might be hard-wired, they habituate if triggered repeatedly (Randlett et al., 2019; Rankin et al., 2009; Thompson and Spencer, 1966). This habituation can be a reduction of the behavioural response, regardless of the evoking stimulus, but can also be selective to the sensory modality (Vogel and Wagner, 2005) or even to the specific stimulus (Finkenstädt and Ewert, 1988; Gutfreund, 2012; Peeke and Veno, 1973; Schleidt et al., 2011). It is essential for animals to learn to ignore innocuous stimuli, but still respond to real threats. Mouse defensive reactions to looming stimuli are habituated by repetition (Salay et al., 2018), but whether the habituation in visually induced fear responses is stimulus selective is not known. Here, we adapted the mouse behavioural paradigm of De Franceschi and colleagues (2016) and passed dark stimuli overhead, similar to birds of prey cruising the sky. We repeated the passing stimuli in several sessions to study whether habituation of the defensive behaviour also occurs in response to this type of stimulus. We investigated whether the defensive behavioural response itself is habituated, or whether the habituation is specific to the stimulus shape or head-centred location in the visual field.

Netherlands Institute for Neuroscience, Royal Netherlands Academy of Arts and Sciences, 1105 BA Amsterdam, The Netherlands.

*Author for correspondence (heimel@nin.knaw.nlw)

(D) S.A.v., 0000-0003-1250-6968; K.S., 0000-0001-9327-151X; L.A.B., 0000-00034370-1301; J.A.H., 0000-0002-5291-4184

Received 4 June 2020; Accepted 30 January 2021

\section{MATERIALS AND METHODS Experimental animals}

Male C57BL/6JRj mice from Janvier Labs (Saint-Berthevin, France) were used. We received the mice at $\mathrm{p} 30$. They were housed in pairs on a normal $12 \mathrm{~h}: 12 \mathrm{~h}$ day/night cycle with food and water available ad libitum. Experiments were performed in the daypart of the cycle. Animals were 5-8 weeks old at the first session. Experiments were performed in accordance with national guidelines and regulations, and approved by the institutional animal care and use committees of the Royal Academy of Arts and Sciences and the Netherlands Institute for Neuroscience.

\section{Experimental setup}

The experimental setup consisted of a rectangular acrylic box (51 cm long, $28 \mathrm{~cm}$ wide, $25 \mathrm{~cm}$ high; Fig. 1A) on a raised platform $(18 \mathrm{~cm})$ with an open top, a transparent bottom and opaque white walls. An LCD display (Dell) matching the dimensions of the box was placed on top of the box to show the stimuli. We filmed the experimental session with a Raspberry Pi Camera outfitted with an $0.4 \times$ wide-angle lens placed underneath the setup. After each experiment, the inside surfaces were cleaned with $50 \%$ ethanol and then water to remove any traces and odours. The entire setup was contained in a sound and light proof behaviour box.

\section{Behavioural experiments}

The experimenter started handling the mice 1-2 weeks after their arrival. The goal of handling the mice was to familiarize them with the experimenter, experimental room and being moved by hand. We handled the mice 2 or 3 times, depending on their behaviour, before placing them for the first time in the behavioural arena for one familiarization session.

Each behaviour session started with a shaping period that lasted for $10 \mathrm{~min}$ for the first session and $5 \mathrm{~min}$ for the subsequent sessions. In this period, mice could see the grey background on the monitor and freely explore the arena to familiarize themselves with the environment. After the shaping period, the experimenter started the first stimulus presentation at the moment the mouse was moving. The stimulus passed once in the middle along the long axis of the screen in about $3 \mathrm{~s}$. We showed 20 stimulus sweeps in each session with roughly $1 \mathrm{~min}$ in between. Each time a sweep was started when the mouse was moving, although because of the response time of the system and the experimenter, the mouse could have reached the wall before the stimulus appeared. The stimulus moved 10 times from left to right on the screen and 10 times in the opposite direction, in random order. In the session where both the habituated stimulus (that had been presented in the previous sessions) and a novel stimulus were presented, each stimulus was shown 10 times, in random order. Occasionally, the total number of presentations was slightly different from 20 by accident. Per session, a mouse was thus about $25-30 \mathrm{~min}$ in the behaviour arena. There were 1-3 days between sessions. 
A

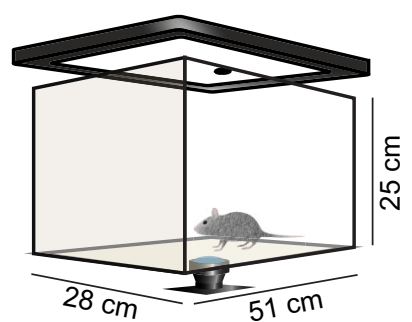

D

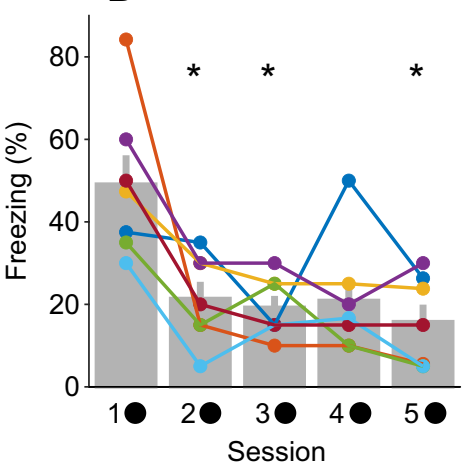

B

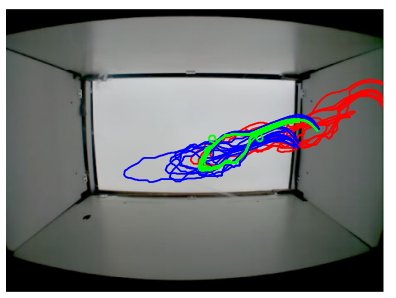

E

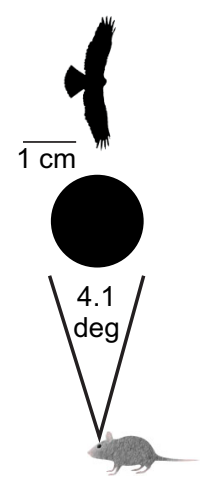

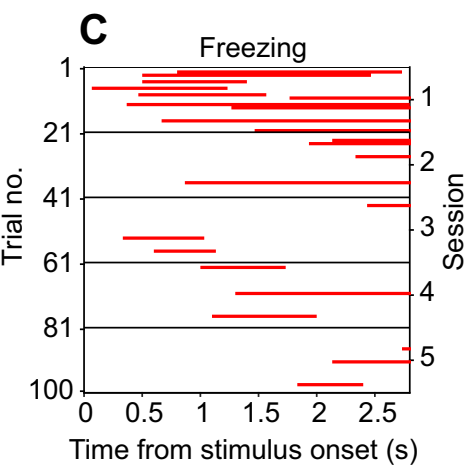

$\mathbf{F}$

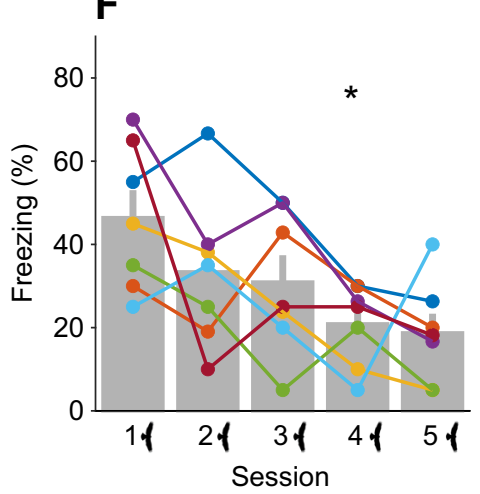

Fig. 1. Mice habituate to stimuli passing overhead. (A) An acrylic glass observation box with a wide-angle camera mounted below and a display mounted on top. (B) Snapshots of the mouse outline every $0.33 \mathrm{~s}$, showing the $3 \mathrm{~s}$ before stimulus onset (red), during the stimulus (green), and the $3 \mathrm{~s}$ after the stimulus disappeared (blue). (C) Freezing periods for the 5 sessions of one mouse (red). (D) The percentage of freezing in response to a disc passing overhead for

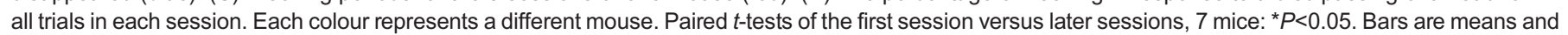
s.e.m. (E) The disc and hawk stimuli. (F) Freezing for sessions with the hawk stimulus. Each colour represents a different mouse (different mice from those in $D$ ). Paired $t$-tests of the first session versus later sessions, 7 mice: ${ }^{*} P<0.05 ;{ }^{* *} P<0.01$.

\section{Stimuli}

We started our experiments with a black disc stimulus of $1.8 \mathrm{~cm}$ diameter (4.1 deg when the disc is directly above the mouse), and a black silhouette of a hawk in flight with a wingspan of $2.4 \mathrm{~cm}$ ( $5.5 \mathrm{deg}$ when overhead). For investigation of the different features, we also used three other stimuli: (1) a small black ellipse roughly matching the hawk's wingspan and surface area (1.6 deg short axis, $4.4 \mathrm{deg}$ long axis when overhead), (2) a small black disc matching the surface area of the hawk and small ellipse (2.5 deg diameter when overhead), (3) a large black ellipse with an aspect ratio matching that of the hawk and surface area matching that of the original black disc (2.5 deg short axis, $6.2 \mathrm{deg}$ long axis when overhead). The stimuli traversed the full long axis of the screen in $3 \mathrm{~s}$ at a fixed speed of $17 \mathrm{~cm} \mathrm{~s}^{-1}$ (39 deg $\mathrm{s}^{-1}$ when passing overhead) along the middle of the short screen axis. The stimulus moved from left to right and right to left in random order. The stimuli were presented using a custom Matlab toolbox built on top of the Psychophysics Toolbox (Kleiner et al., 2007).

\section{Quantification of freezing}

We analysed the video recordings ( 30 frames $\mathrm{s}^{-1}$ ) using a customwritten Matlab program, through which we tracked the movements of mice and stimuli, checking for periods of mouse inactivity around the stimulus presentation. These periods were computed by taking the mean of the absolute difference in pixel intensity (ranging between 0 and 255) of one frame compared with the previous frame in a region extending 100 pixels in all directions around the mouse. The movement signal $M(f)$ in frame $f$ was computed by smoothing this absolute difference in time by taking the mean over a moving window
10 frames $(0.33 \mathrm{~s})$ wide. Next, we computed $M_{\min }$ as the minimum movement occurring during the entire video. Periods of inactivity were defined as contiguous frames where $M(f)<0.3+M_{\min }$ and $\operatorname{abs}(M$ $(f))<0.08$, where $M^{\prime}(f)$ is the frame-wise difference of $M$. This definition and parameters were chosen to make the algorithm agree as much as possible with the human observer estimates of periods of no activity. We marked as freezing an inactivity period of at least $0.5 \mathrm{~s}$, starting during the period that the stimulus was visible. The automated freezing detection still marked some periods as inactivity when mice were not moving in the arena but were grooming or sniffing a corner. We monitored each trial and manually corrected the inactivity periods to remove these false positives.

\section{Analysis of head-relative stimulus position}

We used a custom-written Matlab script to determine the position of the snout and the viewing direction of the mouse from the movie. First, the background was automatically determined from movies by taking the mode for each pixel during the whole session. Next, this background was subtracted from each frame and the result was thresholded. After a number of cycles of image dilation and erosion to connect the thresholded regions, the largest connected remaining component was the mouse. The point in this component that was furthest away from its centre of mass via an internal path was deemed the tail tip. From the tail tip, the two edges of the tail were traced until they separated more than a certain width. This point was the tail base. The snout was the point on the component that was furthest away from the tail base. The vector from the mouse centre of mass to the snout was taken as the approximate head direction. The position from the stimulus was also inferred from the movie. Using 
these values, we computed the location of the stimulus relative to the mouse head and head direction.

To compute the conditional probability that a freeze occurs when the stimulus is in a specific part of visual space, we divided the visual space in sectors in head-relative coordinates. For this purpose, we used two coordinate systems: a 2D horizontal Cartesian system along the screen with one axis along the body axis (used for Fig. 2), and a polar coordinate system, centred at the head of the mouse with one pole directly overhead (used for Fig. S1). Using these coordinate systems, we partitioned each axis individually into an equal number of parts of equal size. The division into sectors was started from a corner placed on the head of the mouse. For example, for Fig. 2C, we divided the Cartesian axes from $-60 \mathrm{~cm}$ to $60 \mathrm{~cm}$ into 12 parts of $10 \mathrm{~cm}$, giving us $12 \times 12=144$ sectors. For each sector $x$, we counted for all mice in all trials of all sessions with only the habituating stimulus, how often the visual stimulus passed through while the mouse was freezing. We also counted for all these trials, how often the visual stimulus passed through each sector $x$, regardless of the state of the animal, and divided this by the total number of traversed sectors to give the probability that the stimulus was in the sector: $P$ (stimulus in $x$ ). Next, we counted how often the visual stimulus was in each sector $x$, when the mouse started freezing, and divided this by the total number of freezes, $P$ (stimulus in $x$ and freezing started). We computed the conditional probability that a freeze started in a specific sector by dividing the freeze start probability by the traversing probability:

$$
\begin{aligned}
& P(\text { freezing started } \mid \text { stimulus in } x)= \\
& P(\text { stimulus in } x \text { and freezing started }) / P(\text { stimulus in } x) .
\end{aligned}
$$

For sectors that the stimulus did not traverse during our recordings (i.e. on the outer rim in Fig. 2E), we set $P$ (freezing started|stimulus in $x$ ) to zero in our graphical representation (Fig. 2F). This probability distribution does not yet reveal anything about the retinotopic location specificity in the habituation. Now, suppose that a mouse was freezing when the stimulus was in sector $x$, and it completely habituated to the stimulus in that sector and would never freeze again when the stimulus was in that sector. In such a case, the total count of freezes while the stimulus was in sector $x$ for one mouse would be 1 or 0 . If the habituation occurred equally for all visual sectors, regardless of which sectors the stimulus traversed, then the mouse could freeze more than once when the stimulus was in a sector. However, even if the habituation was very head centred location specific, choosing a division of visual space in large sectors would result in multiple freezes per sector. In contrast, choosing a division in many small sectors would give a maximum of two freezes, even if habituation was location unspecific, because the odds of stimulus paths crossing
A

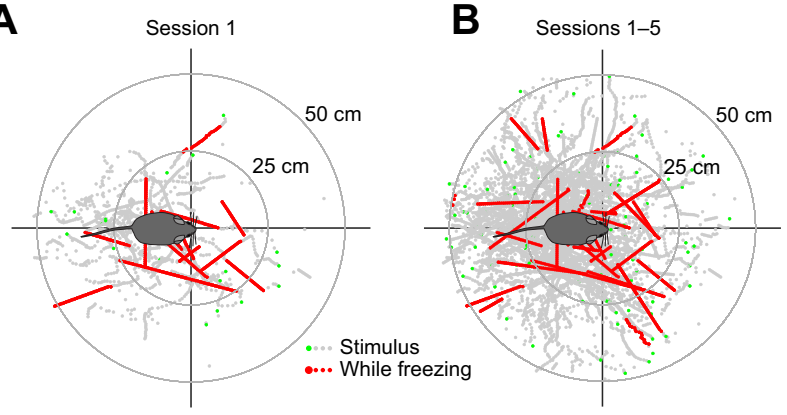

$\mathbf{E}$

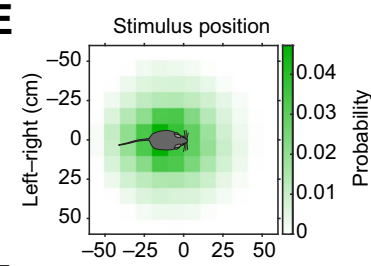

$\mathbf{F}$

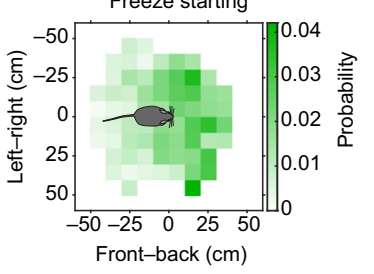

G

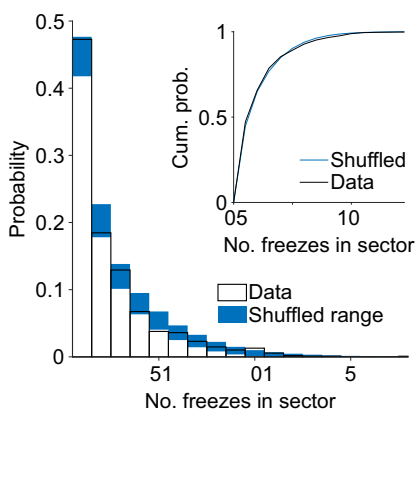

C

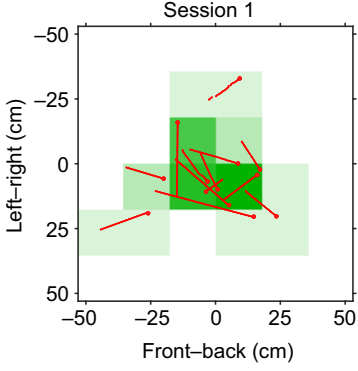

D

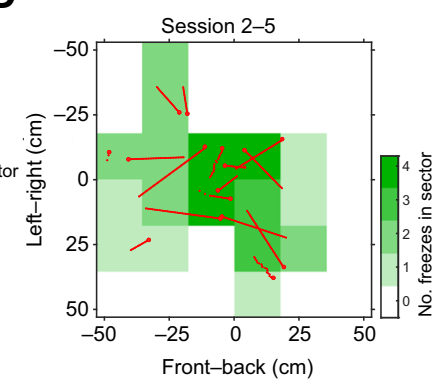

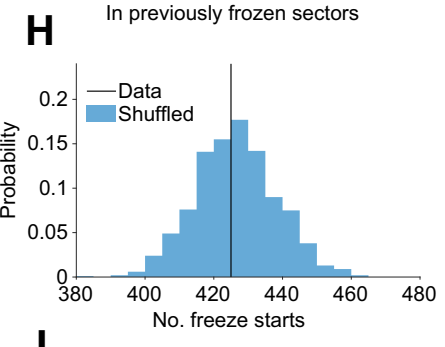

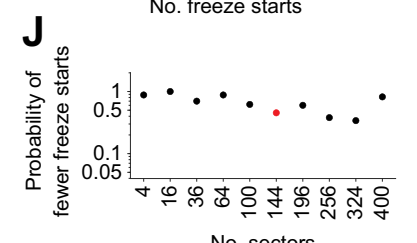

In previously seen sector

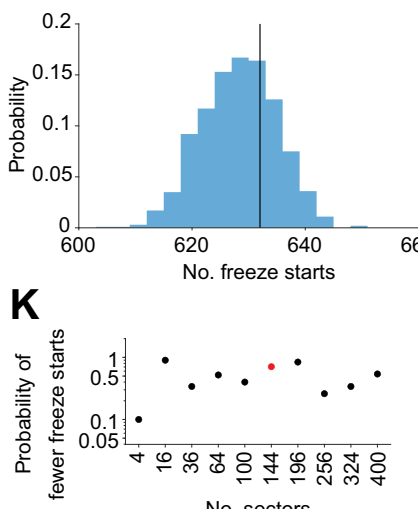

Fig. 2. Habituation is not specific to the head-centred stimulus location. (A) Location of the stimulus relative to the mouse for the first session of an example mouse. The entry of the stimulus is shown in green. Red marks the stimulus trajectory while the mouse was freezing. Mouse is not drawn to scale. (B) Same as in $A$ but for all 5 sessions. (C) Example of division of the overhead visual space in 36 sectors, counting the number of times freezing occurred while the stimulus was in each of these sectors for the data shown in A. The inset (top right) shows a histogram of these numbers. (D) Results for sessions $2-5$ for same mouse as in C. (E) Probability density of the relative stimulus locations during the entire trajectories across all mice. Mouse is not drawn to scale. (F) The conditional probability of a freeze starting for a given stimulus location. (G) Distribution of the number of freezes occurring for each stimulus location for the division in sectors used in $\mathrm{E}$ and $\mathrm{F}$. Blue indicates the range of $2 \mathrm{~s}$.d. below and above the mean for the cases when the freezes were shuffled across mice. The inset shows the cumulative distribution of the data and mean of the shuffled distributions. $(\mathrm{H})$ The number of freeze starts that occurred in a sector that was previously traversed by the stimulus while a mouse was frozen (black line) falls inside the distribution when the freezes are shuffled across mice. (I) Same as $H$ for sectors traversed by the stimulus regardless of whether the mouse was freezing. (J) Fraction of freeze starts in a previously frozen sector of shuffled distribution

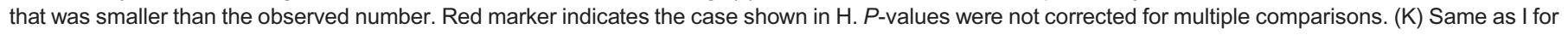
the number of freeze starts in previously seen sectors. Red marker indicates the case shown in I. See also Fig. S1. 
in exactly the same sector more than once become very small. To detect location specificity in the habituation, we therefore used an intermediate sector size and compared the distribution of the number of freezes per sector with the distribution that we got when we generated random freezing data without location-specific habituation. First, we computed the probability distribution of the number of freezes when the stimulus was in each sector per mouse for all sectors in which a freeze occurred at least once (Fig. 2C,G). To compare this observed distribution with a distribution where there is certainly no location-specific habituation, we shuffled the trajectories of trials with freezes across mice and sessions, and randomly picked a point on these stimulus trajectories for the freezing to commence. We computed this distribution for 1000 shuffles, and plotted the mean \pm 2 s.d. of the probabilities in Fig. 2G. We also computed the number of freeze starts in our dataset that occurred in sectors that the stimulus had previously traversed when a mouse was frozen, or in which a stimulus just previously traversed (Fig. 2H,I). This analysis was done across all sessions with only the habituating stimulus. To see whether this number was lower than in the case where experience with specific paths of previous trials cannot have influenced the behaviour, we again shuffled the trials with freeze starts across mice, and randomly picked a point on these stimulus trajectories for the freezing to commence, and recomputed the number of freeze starts in sectors in which the stimulus had previously been, when the mouse was frozen or not. We did this for 1000 shuffles and the resulting distribution, for one specific division of visual space, is plotted in Fig. 2H,I. The results for different divisions are shown in Fig. 2J,K and Fig. S1.

\section{Statistical analysis and sample size}

To see whether an individual mouse froze with a different frequency in different sessions or in response to different stimuli, we used a chi $^{2}$ test. To compare freezing averages of a group of mice between the first and last session with only the habituating stimulus or between the habituated and novel stimuli in the last session, we used paired $t$-tests. For computing the significance of a possible headcentred location-specific effect, we applied 1000 shuffles to the freezing data as described above. Based on the results of Fig. 3B, we computed that 10 mice per group were needed to obtain a similar effect at 0.05 significance level with a likelihood (power) of $85 \%$ for the tests in Fig. 4. By accident, wrong stimuli were shown to 2 mice during one of the sessions. These mice were removed from the results. This led to two groups of 9 mice instead of 10 . With 9 mice, we had a power of $80 \%$ to observe the effect seen in Fig. 3B.

\section{RESULTS}

\section{Mice habituate to stimuli passing overhead}

We placed the mice in an experimental box covered by a monitor and recorded from underneath (Fig. 1A,B). After a short familiarization period, a black disc traversed the screen in about 3 s (De Franceschi et al., 2016). Each session consisted of 20 such passes from right to left or vice versa with a minute between passes. Freezing was scored as the absence of any movement for at least $0.5 \mathrm{~s}$ that started while the stimulus was passing. On average, mice froze in response to $49 \%$ of the stimulus passes (trials) in the first behaviour session (Fig. 1C,D; Movie 1). We repeated this paradigm with the same stimulus for five sessions with 1-3 days between sessions. Mice strongly habituated to the sweeping stimulus (repeated measures ANOVA $P=0.005,7$ mice, Fig. 1D; Movie 2). The percentage of trials in which the mice froze dropped from $49 \%$ in the first session to $16 \%$ in the fifth session (Bonferroni-corrected paired $t$-test $P=0.025)$. Most of this decrease occurred from the first to the second session, when the freezing occurrence dropped to $21 \%$ (Bonferroni-corrected paired $t$-test $P=0.047$ ). The freezing response and its subsequent habituation was not specific to the disc stimulus. We repeated the same test, with naive mice, using a black silhouette of a hawk (Fig. 1E). This also showed a reduction of the responses over sessions (repeated measures ANOVA $P=0.005,7$ mice, Fig. 1F). In the first session, mice froze on $46 \%$ of the presentations (not different from the disc, $t$-test $P=0.78,7$ mice each group). This dropped to $20 \%$ in the fourth session, much smaller than in the first session (Bonferroni-corrected paired $t$-test $P=0.019$ ), and not different from the fifth disc session ( $t$-test $P=0.65)$. The habituation is therefore not due to the artificial nature of the disc stimulus. We did not find a significant difference in the amount of freezing and the habituation to the disc versus the hawk stimulus (two-way ANOVA, effect of session number $P=0.000003$,
A

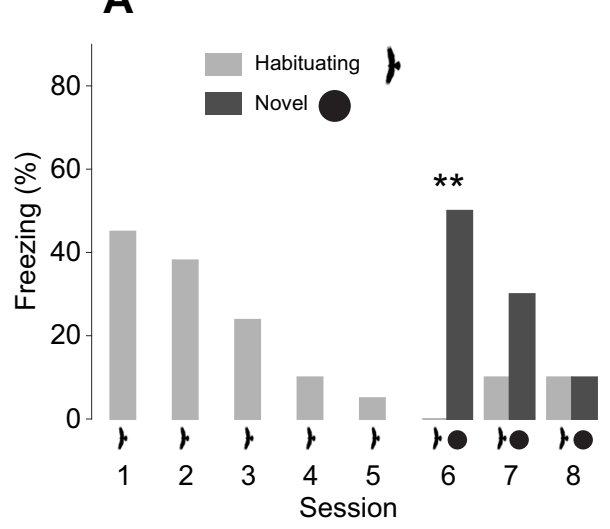

B

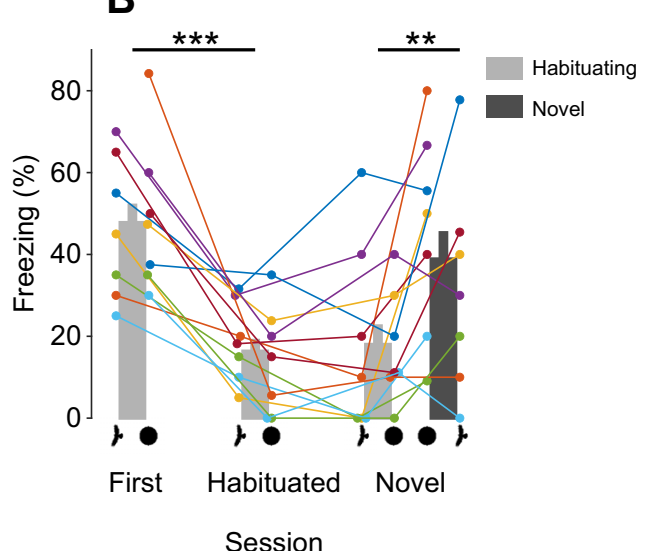

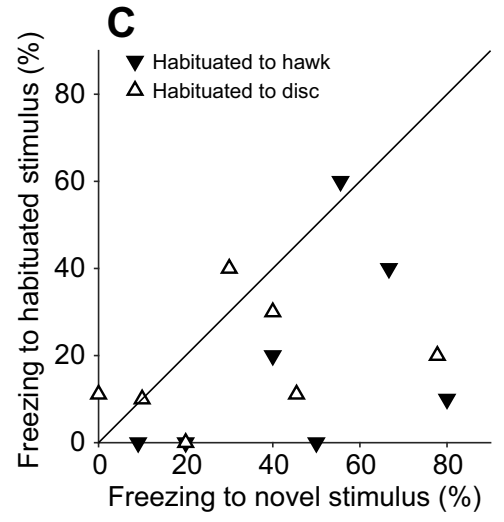

Fig. 3. Habituation is stimulus specific. (A) Example of a mouse freezing in response to a habituating hawk stimulus and a later introduced novel disc stimulus (differences: session 6, chi ${ }^{2}$-test ${ }^{* *} P=0.0098$; session 7 , chi ${ }^{2}$-test $P=0.26$; session 8 , chi ${ }^{2}$-test $P=1$; 10 trials for each stimulus in each session). (B) Freezing in the first session and last habituated session in response to a single stimulus, and freezing in the first session when a novel second stimulus was introduced

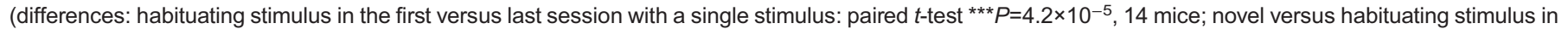
the first test: paired $t$-test $P=0.29$; novel versus habituating stimulus in same session: paired $t$-test ${ }^{* *} P=0.0081$ ). Different coloured lines indicate different mice. Bars are means and s.e.m. (C) Freezing in response to the habituated stimulus versus freezing in response to the novel stimulus in the novel session with two stimuli. Markers show individual mice. 
A

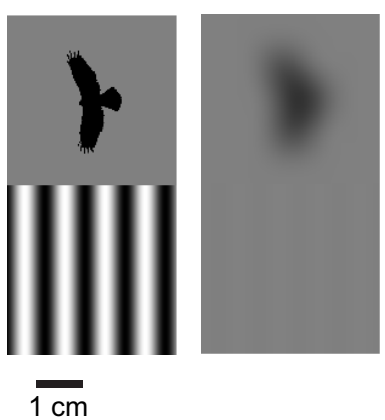

B
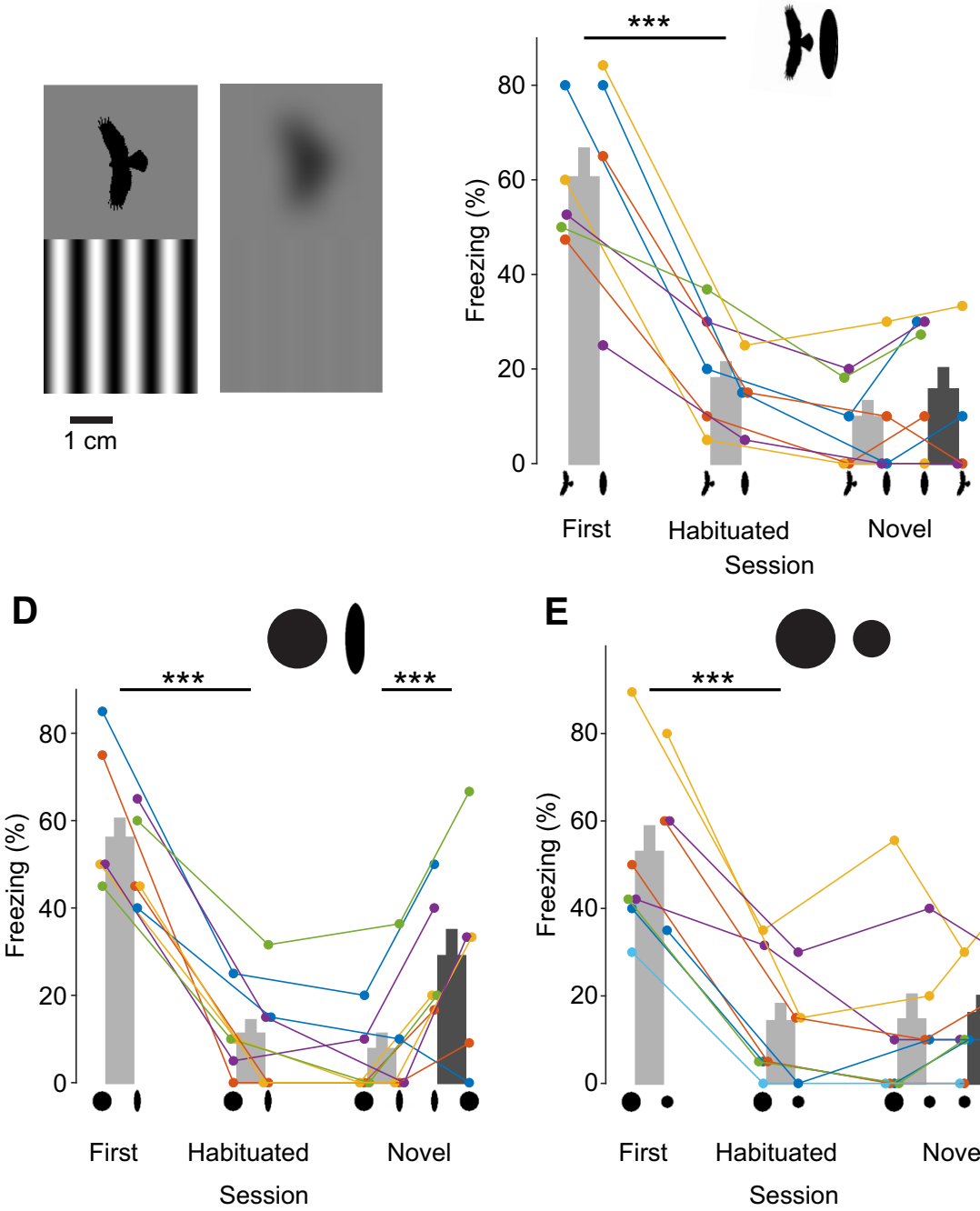

E

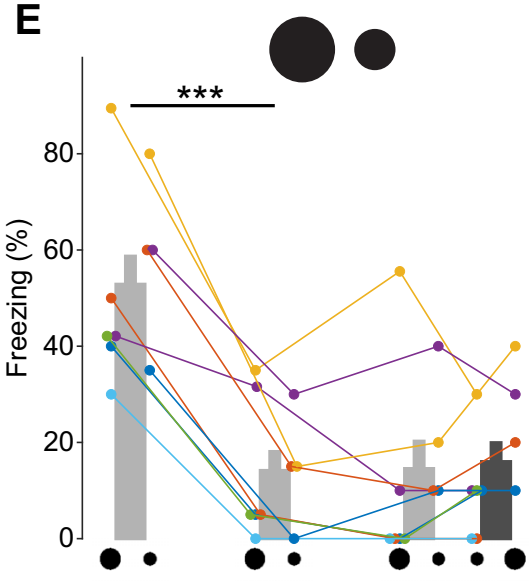

First Session

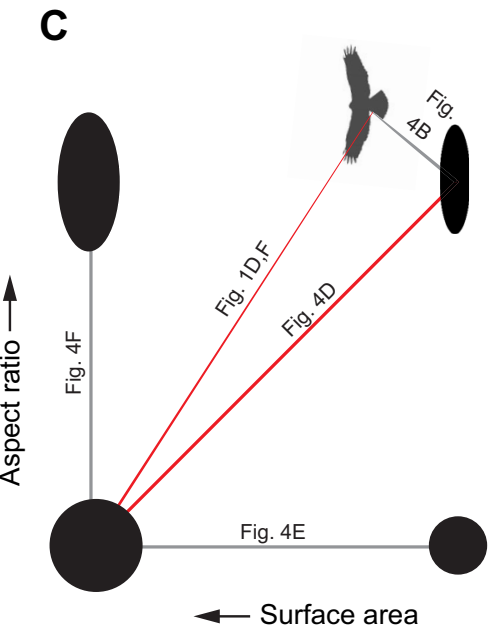

$\mathbf{F}$

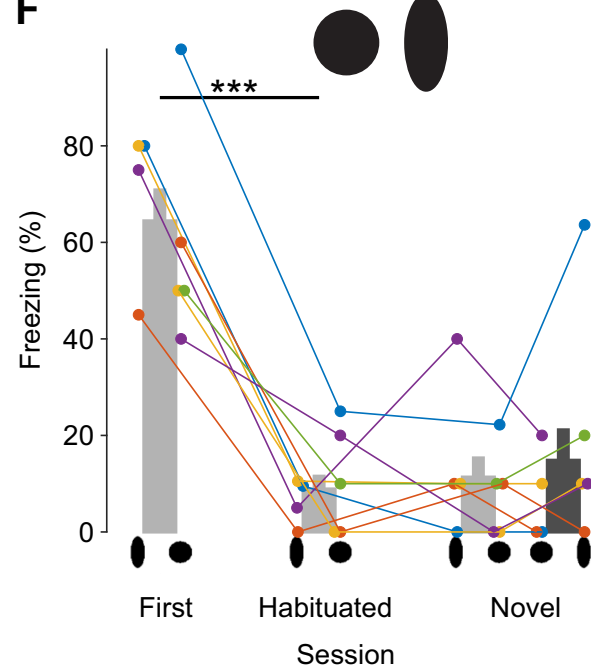

Fig. 4. Habituation is specific to the combination of surface area and shape. (A) Left: hawk stimulus and a sinusoidal grating at the mouse visual acuity limit $\left(0.5\right.$ cycles deg $\left.^{-1}\right)$. Right: the same but after Gaussian blurring that annulled the sinusoidal grating. (B) Freezing in sessions where either a hawk stimulus or an ellipse with an equal surface area and height was used. The animals habituated to the initial stimulus in five sessions (paired $t$-test ${ }^{* * *} P=0.00019,9$ mice). There was no difference between the novel (dark grey bar) and habituated (light grey bar) stimulus in the novel session when the two stimuli were shown interleaved $(P=0.076)$. Bars are means and s.e.m. (C) Stimuli were ellipses differing in surface area or aspect ratio. The hawk is shown next to the closest matching ellipse. The large disc and hawk were used for experiments in the indicated figures. (D) Mice habituated to a large disc or hawk-sized ellipse (paired $t$-test ${ }^{* * *} P=4.9 \times 10^{-6}, 10$ mice), but froze again in response to the introduction of the other stimulus $\left({ }^{* * *} P=0.00081\right)$. (E) Mice habituated in response to a large or small disc (paired $t$-test ${ }^{* * *} P=1.8 \times 10^{-5}, 10$ mice), but did not freeze again in response to the novel stimulus $(P=0.73)$. (F) Mice habituated in response to a large disc and an ellipse with the same area (paired $t$-test ${ }^{* * *} P=1.7 \times 10^{-5}, 9$ mice), but did not freeze again in response to the novel stimulus $(P=0.57)$.

effect of stimulus type $P=0.16$, effect of interaction between session and stimulus $P=0.53$ ).

\section{Habituation is not specific to head-centred stimulus location}

The habituation in the responses of the mice could be due to a diminished response to the stimuli moving overhead at any location, or it could be specific for the retinotopic location at which the stimuli were observed. In the latter case, a stimulus would only lead to freezing once at a specific retinotopic position and then would be ignored when it later appeared at that location again, in the absence of any negative consequences at the first presentation. With repeated presentations, more and more of the retinotopic map would be covered and the amount of freezing would reduce to baseline levels when the whole visual field had been covered. To investigate whether there was evidence in our data to support this hypothesis, we determined the head direction of the mice by measuring the position of the snout relative to their centre of mass. Next, we computed the position of the stimulus relative to the mouse while it was moving across the screen above the mouse, and plotted where it was when the mouse started freezing (Fig. 2A, freezing shown in red). Not surprisingly, we found that over all the sessions the stimulus had been present at many different positions in headcentred coordinates (Fig. 2B) and that the mouse had frozen while the stimulus had been at different positions. Next, to quantify this, we divided the overhead visual space into a number of sectors using different partitions (see Materials and Methods), and for each sector we counted the number of times that a mouse was frozen while the visual stimulus passed through the sector (e.g. Fig. 2C). The example in Fig. 2C shows that the mouse was often frozen when the stimulus was behind its head. This was common to all mice. The reason for this is that mice spend much time investigating the walls and in particular the corners of the box, and were thus pointing away 
from the stimulus (Fig. 2E). This suggests that often a mouse started freezing when the stimulus appeared in the corner of its eye, and then the stimulus moved behind its back. The conditional probability of a freeze starting at a stimulus position relative to the head was, as expected, highest in front of the mouse, where the stimulus could be seen, and low behind the mouse (Fig. 2F). The position and direction of the mouse relative to the stimuli did not appear to change from the first to the later sessions, because the distribution of head-centred stimulus locations was not different in the first session compared with that in the later sessions $\left(\mathrm{chi}^{2}\right.$ test $P=0.11$ ). If the habituation were stimulus location specific, then mice would be less likely to freeze again when the stimulus occurred at a location in the visual field to which the mouse previously froze. This would imply that the distribution of the number of freezes per sector of stimulus locations over all sessions for one mouse would be shifted to a lower number of freezes per sector than if the freezes occurred randomly. We therefore computed the distribution of the number of freezes per sector per mouse over all trials. This distribution was identical to the distribution that we obtained when we shuffled the stimulus trajectories of all trials in which a mouse froze across all mice, indicating that there was no effect of previous experience with a stimulus (Fig. 2G). Furthermore, the number of freeze starts that occurred in head-relative location sectors that the stimulus previously crossed while the mouse was frozen was not different from the number obtained if freezes were shuffled across mice (Fig. 2H). Finally, the number of freeze starts in sectors that the stimulus previously traversed, regardless of whether the mouse froze or not, was not different from the shuffled cases (Fig. 2I). All this suggests an absence of head-centred location-specific habituation. The exact number of freeze starts in Fig. 2G-I depends on the size and shape of the sectors. When taking too large a sector, a finer location-specific habituation could be missed. When taking too small a sectors, our inability to measure the exact gaze direction could remove evidence of location-specific habituation. However, changing the number of sectors (Fig. 2J,K) or changing to a spherical division of visual space (Fig. S1) did not change the conclusion that we found no evidence of stimulus location-specific habituation.

\section{Habituation is stimulus specific}

The absence of head-centred location-specific habituation suggests that the mice habituated to the experience of harmless stimuli passing overhead, regardless of having seen the stimulus before from a specific angle, or perhaps even regardless of having seen the specific stimulus before at all. To check whether mice generalized habituation to all stimuli, in the sixth session we showed the hawk stimulus 10 times and the disc stimulus 10 times, in random order. Mice showed increased freezing again in response to the stimulus that was novel to them (Fig. 3A). In the first session with both the novel and the habituating stimulus, mice froze in $39 \pm 7 \%$ (mean \pm s.e.m.) of the passes of the novel stimulus, while in the same session they only froze in response to $18 \pm 5 \%$ of the passes of the habituated stimulus (paired $t$-test $P=0.0081,14$ mice; Fig. 3B,C). The occurrence of freezing in response to the newly introduced stimulus in the first session with both stimuli was not significantly lower than the freezing that occurred in response to the habituating stimulus in the very first behavioural session $(48 \pm 5 \%$, paired $t$-test $P=0.2$; Fig. $3 \mathrm{~B}$ ). The behaviour of the mice thus clearly indicated a difference between the habituated stimulus and the novel stimulus. There was no difference between the cases where the hawk or the disc was the habituating or novel stimulus (two-way ANOVA $P=0.42,7$ mice in each group).

\section{Habituation is specific to a combination of stimulus surface area and shape}

We were interested in understanding which specific features alert a mouse that a novel stimulus has appeared. Taking into account that a hawk is a natural predator of the mouse, one could hypothesize that elements common to birds like feathers, tail and a beak distinguish the disc from the bird. However, given the limited acuity of mice (Prusky et al., 2000), how much of these details could the mice actually perceive? Indeed, if we simply blur the stimuli to a degree that a grating at the acuity limit $\left(0.5\right.$ cycles $\left.\mathrm{deg}^{-1}\right)$ disappears, the bird starts to look decidedly like a blurred ellipse (Fig. 4A). We therefore hypothesized that the mice would not be able to distinguish between the hawk and an ellipse that matched the hawk in surface area and length along its long axis. Naive mice responded to the ellipse by freezing and again habituated to it over several sessions. However, when we then introduced the original hawk stimulus, they did not freeze significantly more in response to the novel hawk stimulus than they did to the habituating ellipse stimuli. The same was true when we reversed this stimulus order in naive mice. From the first session to the last session with a single stimulus, freezing was strongly reduced (paired $t$-test $P=0.00019,9$ mice; Fig. 4B), but in the session where the second stimulus was introduced, mice did not freeze more in response to the novel stimulus than to the habituated stimulus (paired $t$-test $P=0.076$ ). Therefore, in this freezing assay, mice did not distinguish the hawk and ellipse as different stimuli, indicating that avian features are not enough to make two stimuli distinguishable as overhead threats. Apparently, the hawk and the disc were distinguished by other differences. They also differed in their aspect ratio and by a factor 2.5 in surface area. To separate these two features, we created two new stimuli (Fig. 4C): a smaller disc with the same surface area as the hawk, and a larger ellipse with the same aspect ratio as the hawk and the same surface area as the original disc.

Next, we wanted to reproduce the original experiment of Fig. 3 with the original disc and the small ellipse. We repeated the entire paradigm with naive mice, using the disc and the ellipse stimuli, that were distinguishable by their surface area and aspect ratio (Fig. 4D). Mice again habituated to either stimulus (paired $t$-test $P=4.9 \times 10^{-6}$, 10 mice). When the novel stimulus was introduced after the habituating sessions, the mice clearly increased freezing again in response to the novel stimulus compared with the habituated stimulus $(P=0.00081)$. Mice thus also showed stimulus-specific habituation in this case, demonstrating that they distinguished these two shapes which differed in surface area and aspect ratio but not in the presence of avian features.

Concluding that mice perceived a difference between these stimuli, we were then interested in whether a difference in surface area or aspect ratio alone caused the perception of this difference. To test for an effect of surface area, we habituated naive mice with either the original disc or the smaller disc. While mice habituated to both discs as in earlier experiments (first versus last single-stimulus session, paired $t$-test $P=1.8 \times 10^{-5}, 10$ mice), there was no difference in freezing in response to the disc with the habituated size and the one with the novel size ( $P=0.73$, Fig. $4 \mathrm{E})$.

Finally, we tested whether a difference in aspect ratio alone was sufficient for the stimuli to be distinguished. For this experiment, we used an ellipse matching the original disc in surface area. We repeated the behavioural paradigm in naive mice. Mice showed freezing and habituation in response to both stimuli (different between first and last session with one stimulus, paired $t$-test $P=1.7 \times 10^{-5}, 9$ mice). However, they showed no sign of stimulus-specific habituation to these stimuli (novel versus habituating, $P=0.57$, Fig. $4 \mathrm{~F}$ ). These 
results demonstrate that neither surface area nor aspect ratio alone was the distinguishing feature for mice, but in the case of hawk versus disc, a combination of the two was.

\section{DISCUSSION}

Our results confirm that mice respond to visual threatening overhead stimuli, and show that they adjust their behaviour as their experience with the environment accumulates. In particular, they stop freezing in response to stimuli after repeatedly being exposed to them. This habituation to overhead sweeping stimuli is stimulus feature specific and we identified specific features towards which male mice habituate and the time scale and stimulus presentation regime that drives this habituation. These findings will be useful in future work uncovering mechanisms underlying visually guided behavioural plasticity.

Our experimental paradigm is similar to the 1937 hawk/goose experiment by Lorenz and Tinbergen (detailed in Lorenz, 1939; Schleidt et al., 2011; Tinbergen, 1939). They moved cardboard bird silhouettes over young birds to investigate how birds are able to distinguish between life-threatening raptors and harmless flying creatures. They famously confirmed the hypothesis by Oscar Heinroth that the turkey chicks distinguished raptors from birds such as geese by their short neck. Although this was taken by Tinbergen (1939) as evidence for an innate template, the chicks had seen geese before and stimulus-selective habituation had probably occurred (Schleidt et al., 2011), exactly as we found here in laboratory mice.

We found that mice in their defensive responses generalize over the specific shape and size, but distinguish ellipses that vary in both aspect ratio and size. How the mouse visual system makes this distinction is unclear. The difference (based on the Euclidean norm) in the pixelwise distances between the large disc and small ellipse images, which mice do distinguish, and the large disc and small disc, which they do not distinguish, is only around $5 \%$. If the comparison was performed directly on the retinal images, one would expect much smaller differences in the freezing response to the novel small ellipse and small disc after habituation to the large disc. We only tested ellipses moving in the direction of their small axis. The distinguishable large disc and small ellipse differed by a factor of 2.7 in their size along the drift axis, while the nondistinguishable pairs of large and small disc, and large disc and ellipse only differed by a factor 1.6 in size. A thresholding mechanism on the size difference along this dimension might thus more easily account for the observed data.

The lack of evidence for any specificity to the head-relative location at which the stimulus appeared can also be taken to suggest that the stimulus specificity happens at a more abstract level than the physical image. Mice froze equally in response to stimuli at headrelative positions where stimuli had traversed before and to stimuli at novel locations. We did not measure the pitch and roll of the head, nor did we measure the rotation of the eye within the head. We therefore do not know the exact gaze direction. However, the stimulus paths in, for example, Fig. 2B show that the mouse often did not freeze when the stimulus was at a retinotopic position where it had not previously been. Even if the stimulus trajectories in retinotopic space were highly inaccurate, there were not enough stimulus trajectories during which the mouse was frozen to cover the entire field of view. Some stimulus location-independent habituation must have been occurring. It will be interesting to explore this further, and determine where in the brain this habituation occurs and how the brain is changing such that the freezing response is reduced not only to the experienced stimulus shapes, sizes and locations but also to a combination of shapes, sizes and locations that were not experienced before, while the response is not reduced indiscriminately.

In the rodent, these innate visual defensive responses are thought to be mediated by the superior colliculus (Dean et al., 1989). Its superficial layer receives direct input from the retina, and the information travels from the superior colliculus directly and indirectly to the periaqueductal grey (Evans et al., 2018), where freezing is initiated (Tovote et al., 2016). Location-specific adaptation to a looming stimulus has been observed in the superficial layer of the superior colliculus of head-fixed mice (Lee et al., 2020). The level of abstraction and lack of evidence for any retinotopic specificity in our data for overhead-motion stimuli suggest that the habituating plasticity occurs downstream of the strictly retinotopic superficial layers of the superior colliculus or involves long range connections with these layers. More data from eye tracking and physiological or cellular activity will be needed to determine the locus of the plasticity.

In the stimulus-comparator theory of habituation (Sokolov, 1963), the brain builds a model of the stimulus, and the fit of the stimulus to the model inhibits the stimulus-response pathway. In the mouse, the model building and comparison could be computed in the visual cortex, where stimulus-specific response potentiation occurs (Cooke et al., 2015). Alternatively, we might be witnessing a stimulus-unspecific habituation downstream in the pathway, in combination with a novelty detector, which enhances responses when a new stimulus is detected.

In this study, we have only investigated the behaviour in young male mice. Fear responses and context fear generalization can be different between male and female mice (Borkar et al., 2020; Keiser et al., 2017). Habituation of responses to threats has been reported before in mice (Plappert and Pilz, 2005; Salay et al., 2018), but this instance of stimulus-specific habituation to natural threats provides a new inroad to investigate experience-dependent plasticity in the laboratory. The relevance of this mechanism is not confined to mice. Stimulusselective habituation of defensive responses has been observed in different species and modalities (Deecke et al., 2002). In one of Aesop's fables, written long ago, the fox stopped being frightened by a lion after seeing it twice (Thompson, 2009). In humans, innate defensive behaviours are less easily evoked, but do occur and habituate (Bradley et al., 1993). It is of interest to know how this happens under normal circumstances and in neuropsychiatric conditions, such as autism, ADHD and post-traumatic stress disorder, where habituation is abnormal (Lissek and van Meurs, 2015; McDiarmid et al., 2017).

\section{Acknowledgements}

We thank Mehran Ahmadlou for his help and Christiaan Levelt for sharing equipment and advice.

\section{Competing interests}

The authors declare no competing or financial interests.

\section{Author contributions}

Conceptualization: A.T., J.A.H.; Methodology: A.T., S.A.v.B., J.A.H.; Software: A.T., S.A.v.B., L.A.B., J.A.H.; Investigation: A.T., K.S.; Data curation: J.A.H.; Writing original draft: A.T., J.A.H.; Writing - review \& editing: A.T., S.A.v.B., K.S., L.A.B., J.A.H.; Supervision: J.A.H.; Project administration: J.A.H.; Funding acquisition: J.A.H.

\section{Funding}

A.T. and J.A.H. were supported by Nederlandse Organisatie voor Wetenschappelijk Onderzoek (NWO) Vidi grant 864.10.010

\section{Data availability}

All the scripts for stimulus generation and data analysis are available from GitHub: http://github.com/heimel/InVivoTools. All experimental movies are available on request from the corresponding author. 
Supplementary information

Supplementary information available online at

https://jeb.biologists.org/lookup/doi/10.1242/jeb.230433.supplemental

\section{References}

Borkar, C. D., Dorofeikova, M., Le, Q.-S. E., Vutukuri, R., Vo, C., Hereford, D. Resendez, A., Basavanhalli, S., Sifnugel, N. and Fadok, J. P. (2020). Sex differences in behavioral responses during a conditioned flight paradigm. Behav. Brain Res. 389, 112623. doi:10.1016/j.bbr.2020.112623

Bradley, M. M., Lang, P. J. and Cuthbert, B. N. (1993). Emotion, novelty, and the startle reflex: habituation in humans. Behav. Neurosci. 107, 970-980. doi:10.1037 0735-7044.107.6.970

Cooke, S. F., Komorowski, R. W., Kaplan, E. S., Gavornik, J. P. and Bear, M. F. (2015). Visual recognition memory, manifested as long-term habituation, requires synaptic plasticity in V1. Nat. Neurosci. 18, 262-271. doi:10.1038/nn.3920

De Franceschi, G., Vivattanasarn, T., Saleem, A. B. and Solomon, S. G. (2016) Vision guides selection of freeze or flight defense strategies in mice. Curr. Biol. 26 2150-2154. doi:10.1016/j.cub.2016.06.006

Dean, P., Redgrave, P. and Westby, G. W. M. (1989). Event or emergency? Two response systems in the mammalian superior colliculus. Trends Neurosci. 12, 137-147. doi:10.1016/0166-2236(89)90052-0

Deecke, V. B., Slater, P. J. B. and Ford, J. K. B. (2002). Selective habituation shapes acoustic predator recognition in harbour seals. Nature 420, 171-173. doi:10.1038/nature01030

Eilam, D. (2005). Die hard: a blend of freezing and fleeing as a dynamic defenseImplications for the control of defensive behavior. Neurosci. Biobehav. Rev. 29 1181-1191. doi:10.1016/j.neubiorev.2005.03.027

Evans, D. A., Stempel, A. V., Vale, R., Ruehle, S., Lefler, Y. and Branco, T. (2018). A synaptic threshold mechanism for computing escape decisions. Nature 558, 590-594. doi:10.1038/s41586-018-0244-6

Ewert, J.-P. (1987). Neuroethology of releasing mechanisms: prey-catching in toads. Behav. Brain Sci. 10, 337-368. doi:10.1017/S0140525X00023128

Finkenstädt, T. and Ewert, J.-P. (1988). Stimulus-specific long-term habituation of visually guided orienting behavior toward prey in toads: a14C-2DG study. J. Comp. Physiol. A 163, 1-11. doi:10.1007/BF00611991

Gutfreund, Y. (2012). Stimulus-specific adaptation, habituation and change detection in the gaze control system. Biol. Cybern. 106, 657-668. doi:10.1007/ s00422-012-0497-3

Hoy, J. L., Yavorska, I., Wehr, M. and Niell, C. M. (2016). Vision drives accurate approach behavior during prey capture in laboratory mice. Curr. Biol. 26 3046-3052. doi:10.1016/j.cub.2016.09.009

Keiser, A. A., Turnbull, L. M., Darian, M. A., Feldman, D. E., Song, I. and Tronson, N. C. (2017). Sex differences in context fear generalization and recruitment of hippocampus and amygdala during retrieval. Neuropsychopharmacology 42, 397-407. doi:10.1038/npp.2016.174

Kleiner, M., Brainard, D. and Pelli, D. (2007). What's new in Psychtoolbox-3? Perception 36, 1-16.

Lee, K. H., Tran, A., Turan, Z. and Meister, M. (2020). The sifting of visua information in the superior colliculus. eLife 9, e50678. doi:10.7554/eLife.50678

Lissek, S. and van Meurs, B. (2015). Learning models of PTSD: theoretical accounts and psychobiological evidence. Int. J. Psychophysiol. 98, 594-605. doi:10.1016/j.ijpsycho.2014.11.006
Lorenz, K. (1939). Vergleichende verhaltensforschung. Verh. Dtsch. Zoologischen Ges. Zoologischer Anz. Suppl. 12, 69-102.

McDiarmid, T. A., Bernardos, A. C. and Rankin, C. H. (2017). Habituation is altered in neuropsychiatric disorders-A comprehensive review with recommendations for experimental design and analysis. Neurosci. Biobehav. Rev. 80, 286-305. doi:10.1016/j.neubiorev.2017.05.028

Orioli, G., Bremner, A. J. and Farroni, T. (2018). Multisensory perception of looming and receding objects in human newborns. Curr. Biol. 28, R1294-R1295. doi:10.1016/j.cub.2018.10.004

Peeke, H. V. S. and Veno, A. (1973). Stimulus specificity of habituated aggression in the stickleback (Gasterosteus aculeatus). Behav. Biol. 8, 427-432. doi:10.1016/ S0091-6773(73)80083-5

Plappert, C. F. and Pilz, P. K. D. (2005). Long-term habituation of the startle response in mice evoked by acoustic and tactile stimuli. Behav. Brain Res. 162 307-310. doi:10.1016/j.bbr.2005.03.022

Prusky, G. T., West, P. W. R. and Douglas, R. M. (2000). Behavioral assessment of visual acuity in mice and rats. Vision Res. 40, 2201-2209. doi:10.1016/S0042 6989(00)00081-X

Randlett, O., Haesemeyer, M., Forkin, G., Shoenhard, H., Schier, A. F., Engert, F. and Granato, M. (2019). Distributed plasticity drives visual habituation learning in larval zebrafish. Curr. Biol. 29, 1337-1345.e4. doi:10.1016/j.cub.2019.02.039

Rankin, C. H., Abrams, T., Barry, R. J., Bhatnagar, S., Clayton, D. F., Colombo, J., Coppola, G., Geyer, M. A., Glanzman, D. L., Marsland, S. et al. (2009) Habituation revisited: an updated and revised description of the behavioral characteristics of habituation. Neurobiol. Learn. Mem. 92, 135-138. doi:10.1016/j. nlm.2008.09.012

Salay, L. D., Ishiko, N. and Huberman, A. D. (2018). A midline thalamic circuit determines reactions to visual threat. Nature 557, 183-189. doi:10.1038/s41586018-0078-2

Schleidt, W., Shalter, M. D. and Moura-Neto, H. (2011). The hawk/goose story: the classical ethological experiments of lorenz and tinbergen, revisited. J. Comp. Psychol. 125, 121-133. doi:10.1037/a0022068

Sokolov, E. N. (1963). Higher nervous functions: the orienting reflex. Annu. Rev. Physiol. 25, 545-580. doi:10.1146/annurev.ph.25.030163.002553

Temizer, I., Donovan, J. C., Baier, H. and Semmelhack, J. L. (2015). A visual pathway for looming-evoked escape in larval zebrafish. Curr. Biol. 25, 1823-1834. doi:10.1016/j.cub.2015.06.002

Thompson, R. F. (2009). Habituation: a history. Neurobiol. Learn. Mem. 92 127-134. doi:10.1016/j.nlm.2008.07.011

Thompson, R. F. and Spencer, W. A. (1966). Habituation: a model phenomenon for the study of neuronal substrates of behavior. Psychol. Rev. 73, 16-43. doi:10. 1037/h0022681

Tinbergen, N. (1939). Why do birds behave as they do? (II). In Bird-Lore, Vol. 41, pp 23-30 Macmillan.

Tovote, P., Esposito, M. S., Botta, P., Chaudun, F., Fadok, J. P., Markovic, M. Wolff, S. B. E., Ramakrishnan, C., Fenno, L., Deisseroth, K. et al. (2016) Midbrain circuits for defensive behaviour. Nature 534, 206-212. doi:10.1038/ nature17996

Vogel, E. H. and Wagner, A. R. (2005). Stimulus specificity in the habituation of the startle response in the rat. Physiol. Behav. 86, 516-525. doi:10.1016/j.physbeh. 2005.08.042

Yilmaz, M. and Meister, M. (2013). Rapid innate defensive responses of mice to looming visual stimuli. Curr. Biol. 23, 2011-2015. doi:10.1016/j.cub.2013.08.015 